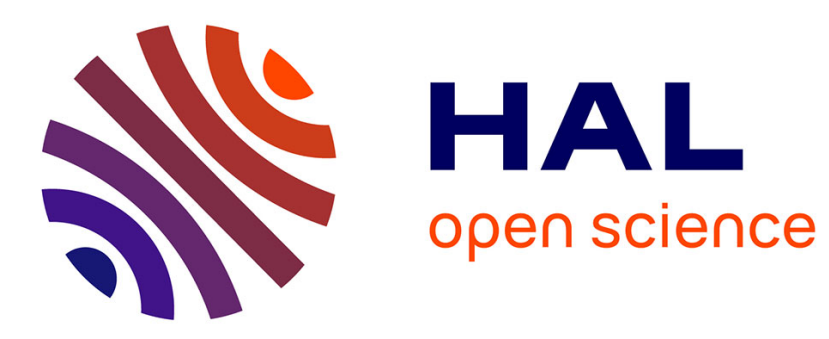

\title{
Towards Urban Traffic Regulation using a Multi-Agent System
}

N Bhouri, F Balbo, S Pinson

\section{To cite this version:}

N Bhouri, F Balbo, S Pinson. Towards Urban Traffic Regulation using a Multi-Agent System. 9th International Conference on Practical Applications of Agents and Multi-Agent Systems (PAAMS11), Mar 2011, SALAMANQUE, Spain. pp.179-188, 10.1007/978-3-642-19875-5_23 . hal-00908083v2

\section{HAL Id: hal-00908083 https://hal.science/hal-00908083v2}

Submitted on 21 Feb 2017

HAL is a multi-disciplinary open access archive for the deposit and dissemination of scientific research documents, whether they are published or not. The documents may come from teaching and research institutions in France or abroad, or from public or private research centers.
L'archive ouverte pluridisciplinaire HAL, est destinée au dépôt et à la diffusion de documents scientifiques de niveau recherche, publiés ou non, émanant des établissements d'enseignement et de recherche français ou étrangers, des laboratoires publics ou privés. 


\title{
Towards Urban Traffic Regulation using a Multi-Agent System
}

\author{
Neila Bhouri ${ }^{1}$, Flavien Balbo ${ }^{1,2}$ and Suzanne Pinson ${ }^{2}$
}

\begin{abstract}
This paper proposes a bimodal urban traffic control strategy based on a multi-agent model. We call bimodal traffic a traffic which takes into account private vehicles and public transport vehicles such as buses. The objective of this strategy is to improve global traffic and reduce the time spent by buses in traffic jams so that buses cope with their schedule. Reducing bus delays is done by studying time length of traffic lights, giving priority to buses, more precisely to buses running late. Regulation is obtained thanks to communication, collaboration and negotiation between the agents of the system. The implementation has been done using the JADE platform. We have tested our strategy on a small network of six junctions. The first results of the simulation are given. They show that our MAS control strategy improves both bus traffic and private vehicle traffic, decreases bus delays and improve its regularity compared to a classical strategy called fixedtime control.
\end{abstract}

\section{INTRODUCTION}

To improve route times of public surface transportation (bus, tramways, shuttles, etc.), cities often use regulation systems at junctions that grant priority to vehicles. These systems are referred as systems equipped with bus priority. The aim of these strategies is to increase the average speed of all vehicles as well as public transport vehicles needed to cross a junction.

The use of these systems is efficient when traffic is light or when they are used to improve a single congested bus route. However, reducing the time of bus journey, although very important for operating a route, is not the primary factor considered by public transport operators whose obligation is to provide the passengers services i.e. keeping interval between buses. In order to take into account the public transport vehicles specificity, TRSS (Transportation Regulation Support Systems) were developed. TRSS systems follow a micro-regulation based approach i.e. modeling the behavior of each bus [1],[2]. One of the weaknesses of these systems is that the private vehicle traffic flow is hardly taken into account. If it is taken into account, this is only as an external parameter that modify the route times of

${ }^{1}$ INRETS/GRETIA, "Le Descartes 2" 2 rue de la Butte Verte, 93166 Noisy Le Grand Cedex. e-mail: neila.bhouri@inrets.fr

${ }^{2}$ University Paris-Dauphine - LAMSADE, Place du Marechal de Lattre de Tassigny F-75775 Paris 16 Cedex, France.

e-mail: balbo@lamsade.dauphine.fr, Suzanne.Pinson@dauphine.fr 
the buses. Another weakness is that traffic light management, which is one of the key factors of traffic jam and bus delays, is not included in the TRSS systems

That's why our objective is to build a traffic control strategy for the bi-modal traffic, able to regulate both the private vehicles traffic and the public transport. Classical control theory to regulate the bi-mode traffic (public transport and private vehicles) is confronted with the modeling problem. Traffic flow can be modeled in a macroscopic or microscopic level. Microscopic modeling is timeconsuming, and it is therefore not well adapted to build real time control strategies for wide urban networks. Macroscopic modeling has been used in by [3] and [4]. However, the macroscopic representation of buses does not allow more than an indirect consideration of the intervals. In these systems the problematic was to reduce the time spent in traffic jams so that buses cope with their bus schedule. In [5] a hybrid model was used : macroscopic modeling for private vehicles and microscopic for the public transport The complexity of the strategy shows the limits of these classic modeling approaches to build a bimodal traffic regulation strategy.

Multi-Agent modeling can be a suitable answer to this scaling problem. We can notice that multi-agent systems are increasingly present in the field of traffic regulation [6], [7]. The problem of traffic lights coordination on the thoroughfares of the route network has been solved in [8], [9]. We already developed a first prototype which shows promising results [10].

The second section focuses on traffic regulation systems and describes our model: the network model and the identification of the agents with a detailed description of agents, their attributes, their objectives, as well as communication and collaboration protocols. The third section provides the first results of the simulation tests carried on the Jade platform. Finally, we conclude in the fifth section.

\section{Network modeling}

In our model, the urban network is represented by an oriented graph $\mathrm{G}=(\mathrm{I}, \mathrm{A})$. The nodes $\{\mathrm{I}\}$ represent the junctions (or intersections) and the arcs $\{\mathrm{A}\}$ represent the lanes that connect the junctions. Two intersections can be connected by one or several arcs depending on the number of lanes on the thoroughfare.

An arc corresponds to a lane. It is characterized by a set of static information (such its length, its capacity, its saturation output which is the maximum output of exits from the given arc) and dynamic information (.the number of vehicles on the arc, the state of the traffic lights at the extremity of the arc: green or red. If the light is green then the vehicles present on the arc can depart).

A junction is specified by the set of the arcs that enter it $E$ and the set of the arcs that leave it $S$. A junction is managed by a set of stages $P$. Each of the stages specifies the list of arcs for which the green light is awarded if the stage is active (see figure 1). 
The network is used by a number of bus routes. Each route comprises the number of buses of the same origin and in the same direction, and which services a number of predefined commercial bus stops at regular time intervals. The time spent by a bus at a commercial stop will be equal to the pre-set time for passengers to mount, plus additional time to regulate the interval, if required.

Fig. 1. Example of a junction with 4 arcs and two stages $\mathrm{P}=\{\mathrm{P} 1, \mathrm{P} 2\}$. $\mathrm{P} 1$ allows for the clearing of the arcs a1 and a3, because the entry flow a1 and a3 can leave the junction at the same green light period. Similarly, P2 clears arcs a5 and a8. The entries and exits of the junction are respectively $\mathrm{E}=\{\mathrm{a} 1, \mathrm{a} 3, \mathrm{a} 5$, $\mathrm{a} 8\}$ and $\mathrm{S}=\{\mathrm{a} 2, \mathrm{a} 4, \mathrm{a} 7, \mathrm{a} 6\}$.

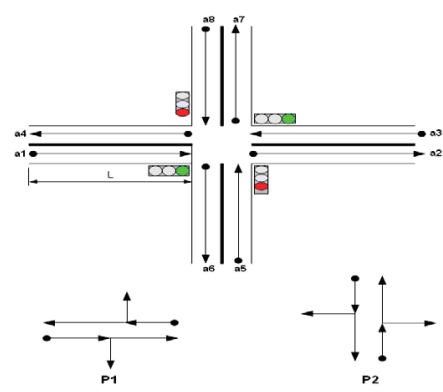

\subsection{Agent modeling}

In order to identify agents and design the MAS we represent an abstraction of the real system; for every entity of the real world is associated an agent in the virtual world to form a Multi-Agent System (MAS). Homogenous agents are called "agent-type". The developed MAS is made up of the following agent-types:

Junction Agent (JA): is the key agent of our architecture. It is in charge of controlling a junction with traffic lights, and of developing a traffic signal plan. The junction agent modifies the planning of the lights according to data sent by approaching buses.

Stage Agent (SA): the traffic signal plan is elaborated thanks to the collaboration of the junction and stage agents. Each SA is expected to determine the optimal green light split to clear the waiting vehicles on the arcs concerned by the stage. Thus, whatever the complexity of the junction is (and its physical configuration), it is managed by a set of stage agents interacting with the junction agent in order to develop a plan of actions for the traffic lights.

Bus Agent (BA): represents a bus in the real world. It circulates from one arc to another, halts at commercial stops, halts at red lights and obeys the instructions of the bus route agent. The objective of each bus agent is to minimize the time spent at traffic lights (i.e. to minimize journey times).

Bus Route Agent (BRA): the bus agent only provides a local view of their environment and, in particular, only the journey covered by the BA. Thus, local optimization carried out by bus agents can have a negative impact on the route, notably on its regularity (i.e. the formation of bus queues). To tackle this problem, we 
propose an agent who has a global view of the route agents, and who can control and modify their behavior in order to guarantee an efficient and regular service.

\subsection{Description of agent behavior}

Bus Agent (BA): In order to minimize the time spent at traffic lights the bus agent interacts with junction agents and its hierarchical superior agent (BRA). All the buses have to provide a regular service and avoid bus queues, in other words, the frequency of buses passing commercial stops must remain stable. To achieve this objective, the BA receives orders from the BRA (for example, stay at the stop for $t$ seconds, if the bus is ahead with respect to the position of the preceding bus).

The BA is composed of a data module, which represents its internal state, and a communication module, which enables exchange with other system agents.

Behavior of a bus agent: Let $\mathrm{t}_{0}$ be the entering time of the bus agent which behaves in the following way:

- On entering arc $i$, the BA retrieves information from the arc (the number of vehicles that precede it, the length, capacity, and exit output of the arc). By using this data, the BA calculates a time-space request, which is transmitted to the JA in order to prevent an eventual stop at the red light at the following junction. The JA then attempts to satisfy the demand (see junction agent below);

- When approaching a stop, the BA informs the associated BRA. The bus route agent then calculates the duration of the regulation interval and its level of priority and sends it to the bus. The bus must wait during the passenger loading time, as well as the potential regulation time, before leaving the stop.

Calculation of a green light request. This calculation is specified by the interval of time during which the green light is granted to the actual arc so that the bus can pass without stopping at the next junction. Let $R$ be the requested interval: $R=\left[t_{b}\right.$, $t_{e}$, with $t_{b}$ and $t_{e}$ be the beginning and ending times of the request interval respectively. The calculation of these times is carried out as follows: the bus enters the arc and finds $\mathrm{N}_{\mathrm{v}}$ vehicles ahead of it, the vehicles move to the traffic lights lane to wait for the green light thus forming a queue of length F In order to continue along its route, the queue of vehicles has to be dispersed before it arrives. The green light should thus be granted at the arc at the instant: $\mathrm{t}_{\mathrm{b}}=\mathrm{t}_{\mathrm{o}}+\mathrm{T}-\mathrm{T}_{\mathrm{F}}$ with $\mathrm{T}$ be the time necessary for the bus to cover the distance between the beginning of the arc and the end of the queue, and $\mathrm{T}_{\mathrm{F}}$ be the time necessary to disperse the queue $\mathrm{F}$.

This request interval $\mathrm{R}$ together with other information (number of bus, its priority, the actual arc of the bus, the next arc to be traveled by the bus) are sent to the JA (at the next junction) who attempts to modify the plan for the lights to satisfy the request. 
Junction Agent (JA): The JA is the key agent of our architecture. The JA supervises the group of stage agents (SA), who collaborate together to establish a plan for traffic lights, which will, on one hand, maximize the capacity of the junction and, on the other hand, attempt to satisfy, as far as possible, the request interval of the bus. The JA is characterized by static and dynamic data.

The static data represents the constraints which characterized the JA. It contains the maximum value of the traffic light cycle (120 seconds). For each cycle, there is an interval of lost time i.e. the period of orange or all red. The all red light is a period during which all the arcs from the same junction have a red light in order to clear the centre of the junction and thus prevent accidents. This fixed period, in conformity with the architecture of the junction, does not depend on the length of the cycle, it is fixed here to a two second period after each stage. It contains also the set of stages of the junction: $\mathrm{P}=\left\{\mathrm{P}_{1}, \ldots, \mathrm{P}_{\mathrm{m}}\right\}$. The set of stages represents the configuration of the junction (the permitted movements and turns). Determining the stages is a task executed offline by the traffic experts.

They are two types of dynamic data, the first is related to the traffic signal plan: it specifies the order of the stages as well as duration of each stage. The second is related to the list of received request data from the bus agents: each request is specified as follows: $R=\left(\mathrm{P}_{\mathrm{i}}, \mathrm{t}_{\mathrm{b}}, \mathrm{t}_{\mathrm{e}}\right.$, Priority), where $\mathrm{P}_{\mathrm{i}}$ is the stage that will allow the passage of the given bus, $t_{b}$ the time when the bus expects to arrive at the traffic light, $t_{e}$, the time when the rear of the bus leaves the arc, and finally 'Priority' is the level of bus priority defined by the bus route agent.

At the end of each cycle, the JA triggers the process of calculating the traffic signal plan for the given cycle. This plan determines the duration of the green light and the ranking of each stage. When the JA receives a request, it records it in the database. The JA decides to accept or to refuse this request at time $t_{b}$. The modification of a traffic signal plan following a priority request by a bus is as follows: 1) Extension of a stage (delay or advance), without exceeding the maximal duration of a stage; 2) Introducing a new stage into the plan.

Calculation of a traffic signal plan. The plan is calculated thanks to the collaboration of the junction agent (JA) and the Stage Agents (SAs). The JA plays the role of a manager in supervising the SAs, which act as participants.

The JA begins by forming a group of collaborators called collab_group including the list of stage agents that needs to be managed. JA initializes the variables: $\mathrm{C}$ $=$ CycleMax, and $\mathrm{t}=0$. Variable $\mathrm{C}$ controls the size of the calculated cycle. JA sends a message to the stage agents to inform them of the protocol initiated to calculate the traffic light plan. JA sends a message request to the agents of the collab_group asking them for the time necessary to clear all the vehicles from their stages, beginning at instant t. Every agent, $i$, of the collab_group calculates its desired green light duration $d_{i}$ and an index that measures the urgency $I_{i}$ of the stage, and sends them to the manager. When the manager receives all the responses, the sum $d$ of durations is calculated. If $\mathrm{d}>\mathrm{C}$ then the manager has to solve a conflict 
(i.e. the size of the cycle exceeds the maximum). Conflict is solved when d previously calculated becomes less or equal to $\mathrm{C}$ : $\mathrm{d} \leq \mathrm{C}$. The manager selects the most urgent stage, that is $\mathrm{P}_{\mathrm{j}}$, its duration is $\mathrm{d}_{\mathrm{j}}$. It sends an accept message to the stage agent in charge of operating this stage; It withdraws the corresponding stage agent of collab_group; It updates the variables $\mathrm{C}=\mathrm{C}-\mathrm{d}_{\mathrm{j}}, \mathrm{t}=\mathrm{t}+\mathrm{d}_{\mathrm{j}}$; finally JA sends request as long as collab_group is not empty.

Conflict resolution. When the sum of green light durations requested by stage agents exceeds the size of the accepted value of the cycle, the manager must restore this sum to the maximal value of the cycle. To achieve a $\Delta t$ reduction, the manager negotiates with the JAs using a Contract net Protocol. The cost of the offer is the number of buses penalized if the stage agent reduces its duration of $\Delta t$.

Stage Agent (SA): This agent has a collection of both static and dynamic data that represents its internal state. The Static data are related to the list of entry arcs, the set of arcs authorized to clear if the stage is active (or green)

Dynamic data are related to 1) the state of the stage: active or inactive; 2) the duration of green light attributed to the stage; 3 ) the starting time of stage execution. 'Active' means that the traffic lights controlling the arcs concerned by this stage are green. The vehicles are therefore authorized to depart.

Behavior of the stage agent. The SA participates to the calculation of the traffic signal plan, and is in charge of fixing the optimal duration of green light for the given stage. When the stage agent is asked about the desired duration of green light by the junction agent, this duration $\mathrm{d}_{\mathrm{i}}$ and an index $\mathrm{I}_{\mathrm{i}}$ that measures the urgency of the stage are computed and transmitted to the junction agent. If the stage agent receives confirmation from the junction agent, the stage agent stops the process. If the stage agent receives a cfp (call for propose) with a cost c, it computes an offer and sends it to the junction agent.

Calculation of the desired duration of the green light

The optimal duration of green light is computed by the following formula:

$$
T=\max _{i=1, \ldots, m}\left\{T_{i}\right\}, \quad T_{i}=w_{i} \frac{\mathrm{N}_{\mathrm{i}}}{\mathrm{D}_{\mathrm{i}}}+\left(1-w_{i}\right) \cdot \frac{\mathrm{N}_{\mathrm{i}} * \mathrm{~L}_{\mathrm{i}}}{\mathrm{C}_{\mathrm{i}} * \mathrm{~V}_{\mathrm{i}}}
$$

with $m$ is the number of entering arcs at this stage, $T_{i}$ the time necessary to clear arc $i, L_{i}$ the length (meter), $V_{i}$ the average speed (meter/second), $N_{i}$ the number of vehicles, $D_{i}$ is the saturation flow and $C_{i}$ the capacity of the arc $i$. The number of vehicles $\mathrm{N}_{\mathrm{i}}$ and the capacity Ci are expressed in private car unit (pcu) which means that all vehicles on the arc are converted to their equivalent on private vehicles, for example a bus is $2.3 \mathrm{pcu}$, depending on its length a track can be 23 or 4 pcu, etc. $\mathrm{D}_{\mathrm{i}}$ is a traffic flow and hence expressed in pcu/second. 
$W_{\mathrm{i}}=\mathrm{N}_{\mathrm{i}} / \mathrm{C}_{\mathrm{i}} \in[0,1]$ is a parameter which indicates the degree of congestion of the arc. When arc is congested, $W_{1}=1$, which means that only the first part of the equation is used.

Urgency index of a stage. In order to award priority to a bus, the urgency index of a stage $\mathrm{j}$ is defined by the fact that the higher the index, the greater the urgency of the stage:

$$
I_{j}=\sum_{i=0}^{m}\left(e^{w_{i}}+e^{b_{i}}\right)
$$

with: $w_{i}$ the parameter indicating the degree of congestion of arc $I, b_{i}$ is the number of buses present on arc I, $m$ is the number of arcs entering via stage $j$ and $\mathrm{e}$ is the Euler constant in our example.

Bus Route Agent (BRA).The role of the route agent is to supervise bus agents so as to prevent a local level regulation and the creation of bus queues. In other words, this agent can modify the behavior of bus agents in two different ways: 1) Directly. by keeping those buses, which are ahead in the plan compared to the preceding ones, at the bus stop for a certain period of time; 2) Indirectly. by modifying bus priorities. This agent has a global view of the route it operates on, and can therefore detect bus queues and react to prevent queue formation

Internal state of the route agent. The route agent encompasses the following data: 1) the set of arcs traveled by the bus on its route; 2) The set of stops on the route: for each stop, its position, and the distance separating it from the next stop; 3 ) The set of buses on the route; 4) The frequency of buses introduced onto the route. For two consecutive stops $A_{i}$ and $A_{j}$, the route agent maintains the journey time $d_{i, j}$ of the last bus. This helps to follow the bus journey and to calculate whether the bus is ahead or late compared to the bus immediately preceding it.

Behavior of the route agent. When a bus agent moves to a stop, the time $t$ taken to cover the distance $\mathrm{L}_{\mathrm{i}, \mathrm{i}-1}$ which separates the two stops $\mathrm{A}_{\mathrm{i}}$ and $\mathrm{A}_{\mathrm{i}-1}$, is transmitted to the route agent. The route agent then compares t to the time $\left(\mathrm{d}_{\mathrm{i}, \mathrm{j}}\right)$ taken by the preceding bus and consequently decides whether the bus is ahead or late. The route agent computes the new priority of the bus agent as well as the length of time the bus should wait at the commercial stop if it is ahead [10]. 


\section{Results}

In order to test our bimodal control strategy, we have developed a Multi-Agent System prototype on the JADE ${ }^{1}$ platform (Java Agent Development Framework). JADE offers Java middleware based on a peer-to-peer architecture with the overall aim to provide a runtime support for agents (JADE, 2009).

We have tested the strategy on a small network of six intersections (figure 7):

- The distance between two adjacent junctions belongs to [200,400] meters.

- Each section comprises one or two lanes.

- The saturation flow, which is the maximum exit output of the arcs, is identical for each arc and equal to 0.5 vehicles/second.

- At each entry onto the network we have installed a source that generates vehicles at a frequency $\mathrm{F} \in[4 \mathrm{~s} \ldots 10 \mathrm{~s}]$.

- Some of the junctions have two stages and the others have three stages.

- Two buses enter the network. For Bus 1, the frequency of the generated buses is 80 seconds and 180 seconds for Bus 2.

We have compared the developed MAS strategy to a fixed time strategy with 30 seconds for each stage. We have run the simulation with these two strategies and for half-hour simulation time.

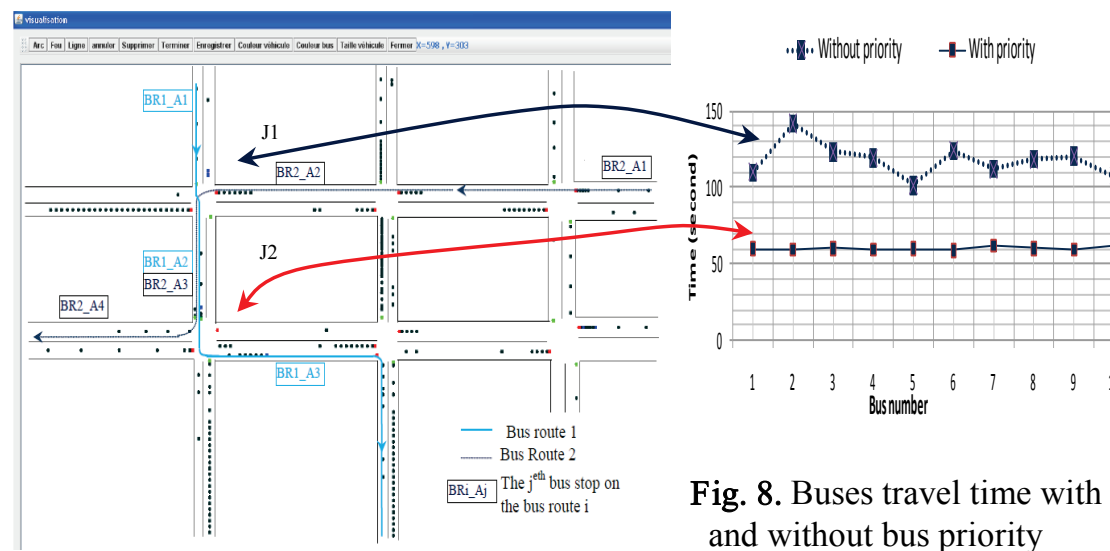

Fig. 7. The simulated network and without bus priority

Figure 8, depicts buses travel time : the higher curve shows buses travel time between the stops BR1_A1 and BR1_A2 when buses do not request priority from the junction $\mathrm{J} 1$; the lower curve show buses travel time between the two bus stops

1 jade.tilab.com/ 
BR1_A2 and BR1_A3 when buses are asking for priority at junction J2. We can notice buses travel time improves and becomes very regular when bus priority is taken into account.

Figure 9 gives results of the two strategies for very heavy traffic conditions. Figure 9.a shows recorded delays for buses with the two control strategies and figure 9.b shows the same kinds of curves for private vehicles.

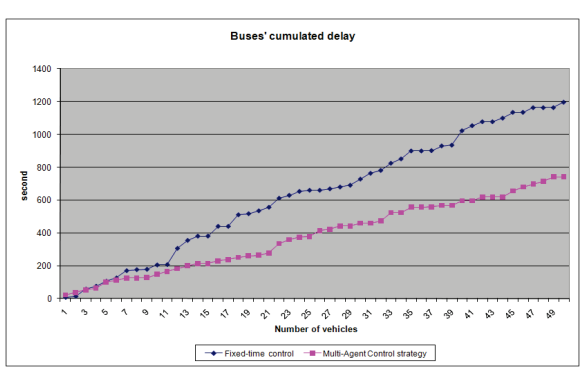

Fig. 9.a. Buses cumulated delays

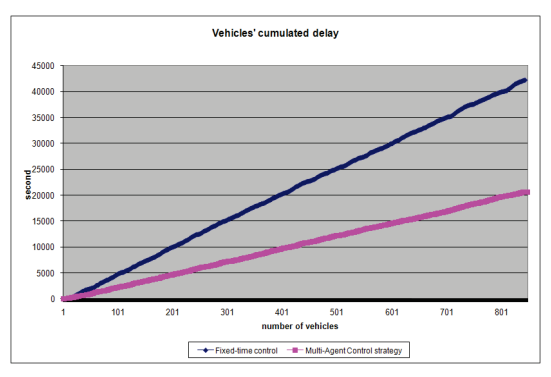

Fig. 9.b Private vehicles cumulated delays

These delays correspond to the sum of time lost by all buses (resp. vehicles) at stops on the traffic lights. As shown on figure 9, the MAS strategy improves both traffic of buses and traffic of private vehicles. As we can see, there is a decrease of $38 \%$ on lost time spent by buses on traffic light; for the private vehicles, we got a decrease of about $51 \%$.

\section{CONCLUSION}

In this paper, we have developed a bimodal traffic control strategy based on a multi-agent system. It takes into account two transportation modes: public transportation i.e. buses and private vehicles. The originality of this strategy is the application of the new information and agent technologies, the entities representing the urban network can communicate among themselves and negotiate in order to solve traffic regulating problems. First, we have shown that classical methods of control systems of traffic regulation present several weaknesses: at a macroscopic level, they do not take into account mixed traffic and does not allow for the regulation of intervals between buses Furthermore computations at a microscopic level are time-consuming specially for regulating large networks. In a second part, we have presented the multi-agent strategy that computes traffic signals plans based on the actual traffic situation and on the priority needed by the buses. The priority is given to those buses that do not deteriorate the intervals between the vehicles on 
the same route. In the third part, we have run a simulation prototype on the JADE platform. Comparison between buses travel time with and without bus priority shows the capacity of the priority method we developed to improve both the travel time and the regularity of buses. Results also show that this bimodal MAS strategy improves conditions of global traffic and reduces bus delays. More work should be done: a more realistic network should be defined in the simulation run and more validation and more testing should be undertaken with the definition of several indicators.

\section{REFERENCES}

1. Balbo, F, Pinson, "Using intelligent agents for Transportation Regulation Support System design", Transportation Research Part C: Emerging Technologies, Volume 18, Issue 1, February 2010, Pages 140-156 Information/Communication Technologies and Travel Behaviour; Agents in Traffic and Transportation.

2. Cazenave T., Balbo F., Pinson S., 2009, "Monte-Carlo Bus Regulation", $12^{\text {th }}$ Int. IEEE ITSC'09, October 3-7, St. Louis, MO, USA pp. 340-345.

3. Bhouri, N. Lotito P., 2005. An intermodal traffic control strategy for private vehicle and public trans-port. 10th Euro Working Group on Transportation, Poznan, Pologne.

4. Bhouri, N., 2009, Constrained Optimal Control strategy for multimodal urban traffic network. IFAC Workshop on Control Applications of Optimization (CAO'09), Finland, May.

5. Kachroudi, S. Bhouri N., 2009. A multimodal traffic responsive strategy using particle swarm optimization. 12th IFAC Symposium. Redondo Beach, California, USA.

6. Bazzan A. L. C. 2008. "Opportunities for multiagent systems and multiagent reinforcement learning in traffic control", Springer Science+Business Media, LLC

7. Mailler R., Lesser V, 2004. "Solving distributed constraint optimization problems using cooperative mediation". In Third Int. Joint Conference on Autonomous Agents and Multiagent Systems, pages 438-445. IEEE Computer Society.

8. De Oliveira D, Bazzan A.L. and Lesser V, 2005. Using Cooperative Mediation to Coordinate Traffic Lights, AAMAS'05, July 2529, Utrecht, Netherlands.

9. Roozemond D. A., 2001. "using intelligent agents for pro-active, real-time urban intersection control”, European Journal for Operationel Research 131. pp 293-301.

10. Bhouri, N., Haciane S. and Balbo F., 2010. "A Multi-Agent System to Regulate Urban Traffic: Private Vehicles and Public Transport. 13th IEEE-ITSC, Portugal, 19 -22 September. 